\title{
Natürliche Radioaktivität im Grundwasser - neue Parameter und Herausforderungen für die Trinkwasserversorgung
}

\author{
Anita Erőss ${ }^{1} \mathbb{D}$ \\ Eingegangen: 4. März 2020 / Überarbeitet: 4. März 2020 / Online publiziert: 13. März 2020 \\ ๑) Springer-Verlag GmbH Deutschland, ein Teil von Springer Nature 2020
}

Die Beschaffenheit des Grundwassers entwickelt sich kontinuierlich durch Wasser-Gesteins-Wechselwirkungen entlang von Fließwegen. Die natürliche geologische und geochemische Umgebung kann dem Grundwasser dabei nicht nur seinen Mineralstoffgehalt einschließlich essenzieller Spurenelemente verleihen, sondern durch einen Überschuss bestimmter Elemente auch zu unerwünschten oder toxischen Eigenschaften führen. Grundwasser kann verschiedene natürliche Alpha- (U-238, U-234, Th-232, Ra-226 und Po-210) und Betastrahler (K-40, Ra-228 und $\mathrm{Pb}-210)$ enthalten, von nicht nachweisbaren Werten bis hin zu Konzentrationen, die für die menschliche Gesundheit bedenklich sind. Die Strahlenbelastung durch das Trinken von aus Grundwasser gewonnenem Trinkwasser kann insbesondere von Alphastrahlung emittierendem Uran, Radium und Radon herrühren.

Hinsichtlich des Trinkwassers sind die Vorschriften für den Schutz der menschlichen Gesundheit in Bezug auf radioaktive Stoffe in der Richtlinie 2013/51/EURATOM festgelegt. In vielen Ländern wird Mineralwasser jedoch als Nahrungsmittel behandelt und ist daher weniger reguliert als Trinkwasser. Daher ist unter den Qualitätsparametern von Mineralwasser der natürliche Radioisotopengehalt weniger bekannt. Der zunehmende Verzehr von abgefülltem Mineralwasser und von aromatisierten Getränken (einschließlich Mineralwasser mit Geschmack) erfordert jedoch eine sorgfältige Bewertung dieses Aspekts, da diese Produkte hauptsächlich auf Grundwasserressourcen angewiesen sind.

Aufgrund der relativ geringen Kosten und der Einfachheit werden meistens die Gesamt-Alpha- und Gesamt-

Anita Erôss

anita.eross@geology.elte.hu

1 József \& Erzsébet Tóth Stiftungslehrstuhl für Hydrogeologie, Abteilung Geologie, Institut für Geographie und Geowissenschaften, Naturwissenschaftliche Fakultät, Eötvös Loránd Universität, Pázmány Péter s. 1/c, 1117 Budapest, Ungarn
Beta-Aktivitätskonzentrationen zur Charakterisierung der Radioaktivität im Trinkwasser als Screening-Verfahren verwendet. Diese Methode wird in den meisten Ländern in nationalen Vorschriften im Zusammenhang mit der neuen Trinkwasserqualitätsrichtlinie in Bezug auf Radioaktivität umgesetzt. Es wurden jedoch viele Unsicherheiten bei dieser Methode festgestellt, die entweder auf analytische Verfahren oder auf natürliche Eigenschaften der Wasserprobe zurückzuführen sind (z.B. gleichzeitiges Auftreten mehrerer Radioisotope, hoher gelöster Stoffgehalt, hoher Nitratgehalt, hoher Eisengehalt). Dies wurde auch durch einen Ringversuch europäischer Messlaboratorien bestätigt, der vom Institut für Referenzmaterialien und -messungen der Gemeinsamen Forschungsstelle (JRC-IRMM) der Europäischen Kommission organisiert wurde. Die von 71 Teilnehmern eingereichten Ergebnisse wichen teils um mehr als zwei Größenordnungen von den Referenzwerten $\mathrm{ab}$, unabhängig von den verwendeten Messmethoden. Dies deutet darauf hin, dass die auf Gesamt-Alpha- und GesamtBeta-Aktivitätskonzentrationen basierenden Methoden in der Gesetzgebung überarbeitet werden müssen. In manchen europäischen Ländern (z.B. Österreich, Schweiz) werden diese Methoden aufgrund ihrer Nachteile und Unzuverlässigkeit nicht mehr für die Trinkwasserbewertung verwendet. In Ländern, in denen diese Methoden für die Umsetzung der Richtlinie verwendet werden, besteht ein ernstes Problem, dass Entscheidungen für Wasserversorgungssysteme auf der Grundlage unzuverlässiger Ergebnisse aus Gesamt-Alpha- und Gesamt-Beta-Messungen getroffen werden. Falsche positive und falsche negative Ergebnisse überschätzen oder unterschätzen die gesundheitlichen Auswirkungen des Trinkwasserverbrauchs. Nur eine nuklidspezifische Analyse liefert eine zuverlässige Schätzung, ob der Trinkwasserverbrauch zu einer jährlichen Dosis von mehr als 0,1 Millisievert (mSv) führt oder nicht. Darüber hinaus liefert nur eine nuklidspezifische Analyse relevante Erkenntnisse über den Zusammenhang zwischen Geologie, Grundwasserfließsystemen und dem Vorkommen natürlicher Radionuklide im Grundwasser. Hier bekommt die Hydrogeologie eine wichtige Rolle. Um 
den Ursprung der gemessenen Werte zu erklären und Lösungsmöglichkeiten für das Wasserwerk bereitzustellen, ist das Verständnis der Grundwasserfließsysteme von zentraler Bedeutung. Grundwasserströmung kann eine erhöhte natürliche Radioaktivität generieren, selbst wenn die Quelle weit entfernt und/oder nicht signifikant ist, denn die Akkumulation durch kontinuierliche Grundwasserströmung kann beträchtliche Radioaktivität erzeugen. Auch Ausfällungen in Leitungssystemen können als kontinuierliche Quellen fungieren. Ein anderes wichtiges Problem kann bei stark instationären Wasserressourcen auftreten, wie z.B. Wasserversorgungssysteme am Flussufer. Hier können die gemessenen Werte stark vom Zeitpunkt der Probennahme abhängen, insbesondere vom Wasserstand im Fluss, der das Mischungsverhältnis zwischen Oberflächengewässer und Grundwasser und damit die Wasserqualität bestimmt.

Radioisotope können auch als natürliche Tracer für das Verständnis von Grundwasserfließsystemen eingesetzt werden. Aufgrund ihres unterschiedlichen geochemischen Verhaltens ist ihr Auftreten jeweils nur für einzelne Segmente von Fließsystemen typisch, die durch bestimmte geochemische Bedingungen charakterisiert sind. So ist Uran im oxidierenden Milieu mobil, insbesondere in Grundwasser mit nahezu neutralem $\mathrm{pH}-$ Wert, hoher Alkalinität und/oder hohem Kolloidgehalt. Radium ist dagegen unter reduzierenden und sauren Bedingungen mobil und tritt typischerweise in Thermalwasser auf. Fließsysteme unterschiedlicher Ordnungen liefern zu regionalen Austrittsgebieten Wasser mit unterschiedlicher Temperatur, chemischer Zusammensetzung und unterschiedlichem Redox-Zustand. Dies ermöglicht die Verwendung von Radium und Uran zur Identifizierung von Mischungsprozessen und -komponenten, z. B. die Quantifizierung der Mischungskorrosion als Höhlenbildungsprozess in Karstgrundwasserleitern. In solchen Fällen können Uran und Radium gute Alternativen zu den stabilen Isotopen von Sauerstoff und Wasserstoff sein. Radionuklidmessungen sind daher nützlich, um die hydrogeologische Forschung und das Verständnis von Fließsystemen zu unterstützen.

Nuklidspezifische Radionuklidforschung und Hydrogeologie können somit Hand in Hand gehen, und Hydrogeologen können dabei behilflich sein, die vom Wasserwerk gemessenen Werte besser zu verstehen und so eine sichere Trinkwasserversorgung zu unterstützen. 